\title{
BMJ Open Senior high-cost healthcare users' resource utilization and outcomes: a protocol of a retrospective matched cohort study in Canada
}

\author{
Sergei Muratov, ${ }^{1,2}$ Justin Lee, ${ }^{1,3,4,5}$ Anne Holbrook, ${ }^{1,4} \mathrm{~J}$ Michael Paterson, ${ }^{6}$ \\ Jason Robert Guertin, ${ }^{7,8}$ Lawrence Mbuagbaw, ${ }^{1}$ Tara Gomes, ${ }^{6,9}$ Wayne Khuu, ${ }^{6}$ \\ Priscila Pequeno, ${ }^{6}$ Andrew P Costa, ${ }^{1,10}$ Jean-Eric Tarride ${ }^{1,2,11}$
}

To cite: Muratov S, Lee J, Holbrook A, et al. Senior high-cost healthcare users' resource utilization and outcomes: a protocol of a retrospective matched cohort study in Canada. BMJ Open 2017;7:e018488. doi:10.1136/ bmjopen-2017-018488

- Prepublication history and additional material for this paper are available online. To view these files, please visit the journal online (http://dx.doi org/10.1136/bmjopen-2017018488).

Received 3 July 2017

Revised 1 November 2017

Accepted 24 November 2017

CrossMark

For numbered affiliations see end of article.

Correspondence to

Mr. Sergei Muratov;

muratos@mcmaster.ca

\section{ABSTRACT}

Introduction Senior high-cost users (HCUs) are estimated to represent $60 \%$ of all HCUs in Ontario, Canada's most populous province. To improve our understanding of individual and health system characteristics related to senior HCUs, we will examine incident senior HCUs to determine their incremental healthcare utilisation and costs, characteristics of index hospitalisation episodes, mortality and their regional variation across Ontario. Methods and analysis A retrospective, population-based cohort study using administrative healthcare records will be used. Incident senior HCUs will be defined as Ontarians aged $\geq 66$ years who were in the top $5 \%$ of healthcare cost users during fiscal year 2013 but not during fiscal year 2012. Each HCU will be matched to three non-HCUs by age, sex and health planning region. Incremental healthcare use and costs will be determined using the method of recycled predictions. We will apply multivariable logistic regression to determine patient and health service factors associated with index hospitalisation and inhospital mortality during the incident year. The most common causes of admission will be identified and contrasted with the most expensive hospitalised conditions. We will also calculate the ratio of inpatient costs incurred through admissions of ambulatory care sensitive conditions to the total inpatient expenditures. The magnitude of variation in costs and health service utilisation will be established by calculating the extremal quotient, the coefficient of variation and the Gini mean difference for estimates obtained through multilevel regression analyses.

Ethics and dissemination This study has been approved by Hamilton Integrated Research Ethics Board (ID\#1715-C). The results of the study will be distributed through peer-reviewed journals. They also will be disseminated at research events in academic settings, national and international conferences as well as with presentations to provincial health authorities.

\section{INTRODUCTION}

Societies worldwide are facing a demographic shift towards a growing proportion of seniors, defined as people aged 65 years and older. ${ }^{1}$ In 2015, the proportion of seniors in Canada, for
Strengths and limitations of this study

- Focusing on incident senior high-cost users (HCUs) and comparing them with non-HCUs in a longitudinal study allows for scrutiny of the factors that are associated with the transition from non-HCU to HCU and for identification of opportunities of proactive preventive management approaches.

- The comparative nature of the study with a matched cohort design reduces bias due to confounding.

- This study is subject to the limitations inherent in observational design and the use of health administrative databases.

- The study is limited by the period of observation of 1 year before and 1 year after becoming HCU for most of the variables.

instance, exceeded the proportion of young people (ie, $<15$ years of age) for the first time in history. ${ }^{2}$ Seniors account for $46 \%$ of the national public healthcare expenditures in Canada. ${ }^{3}$ This proportion is likely to increase due to the continued ageing of the population, therefore putting additional pressure on the government's resource allocation decisions in the coming years. The high-cost users (HCUs) of health services, ${ }^{45}$ commonly defined as individuals in the highest $5 \%$ of total expenditures, are often seniors. Senior HCUs are estimated to represent $60 \%$ of all HCUs in Ontario, Canada's most populous province. ${ }^{4}$ Consistent with findings from other jurisdictions, ${ }^{67}$ a recent Ontario study indicated that $5 \%$ of senior HCUs consume $44 \%$ of the total measured public healthcare expenditures by the seniors in the province. ${ }^{8}$

A number of demographic and clinical characteristics of the senior HCUs have been described internationally and in Canada: high level of comorbidities, functional impairment and poor social supports at home. ${ }^{8-10}$ However, 
many individual and health system characteristics related to senior HCUs are still poorly understood, particularly in the context of their subpopulations. As such, many disease management programmes as well as research efforts focus on persistent HCUs, that is, those who retain their HCU status in subsequent years. ${ }^{81112}$ This practice ignores the fact that 'new', or incident, HCUs have historically accounted for more than $50 \%$ of all the cases annually, including those among senior patients. ${ }^{813}$ Incident senior HCUs may have different characteristics than prevalent HCUs, and more focus on incident HCUs will allow for scrutiny of the factors that influence the transition from non-HCU to HCU.

Filling gaps in our understanding of this HCU subgroup is especially important at a time when policy makers internationally are targeting interventions for senior HCUs such as complex case management and care coordination models. ${ }^{8} 121415$ To inform policy making in identification of opportunities to prevent transition to the HCU status or to improve existing programmes, a closer inquiry is required into the incident senior HCUs in terms of their incremental healthcare utilisation and costs, characteristics of their hospitalisation episodes, including the economic impact of individual conditions, and regional variation in main outcomes.

\section{Incremental costs among incident HCUs}

The magnitude of incremental healthcare utilisation and costs attributable to becoming a HCU is unknown. HCU research in Canada and elsewhere has been conducted predominantly on prevalent HCU cohorts using cross-sectional designs. ${ }^{47816-18}$ These studies provide valuable information on comparisons, for example, of the 1 year costs of HCUs compared with non-HCUs. However, these methods do not explore the change in outcomes associated with becoming a new HCU beyond secular trends in outcomes over time, thus missing the contribution of HCU status. In addition, no study to our knowledge has compared the characteristics, costs and outcomes of incident HCUs to a matched cohort of non-HCUs, which would provide a more detailed assessment of the distinguishing features of HCU status. Finally, HCU-related research with a system-wide approach is still limited ${ }^{4}$ as studies have largely focused on acute care (eg, hospitalisations, emergency care and physicians) and have left out other important care categories such as long-term care, rehabilitation and medications. Recently, a population-based study conducted in Ontario, Canada, took a 1 year look at the cost distribution across a wider range of health sectors among HCUs, including seniors. ${ }^{8}$ Although it was applied to prevalent HCUs and was not intended to provide a detailed characterisation of the study population and a comparison with non-HCUs, we will be building on their work by using the same cost algorithm.

\section{Analysis of hospitalisation episodes among senior incident HCUs compared with non-HCUs}

The majority ( $>90 \%$ in some studies) of senior HCUs have at least one hospital admission in the year they reach HCU status. ${ }^{13}$ Considering that hospitalisation costs among HCUs may account for almost two-thirds of direct medical costs, ${ }^{4}$ it is important to better understand the characteristics associated with hospitalisations among incident HCUs. To date, much of the literature on risk factors and interventions to prevent hospitalisation has focused on hospital readmissions. ${ }^{19-22}$ However, as opposed to younger adults in whom hospitalisations often occur due to a sudden event (eg, trauma) that often resolves without serious permanent cost or care implications, ${ }^{23}$ readmissions in senior patients, especially HCUs, may signal a deterioration in health status and mark a point where management interventions are less likely to be effective in preventing recurrent hospitalisations. ${ }^{12}$ Therefore, focusing on the index hospitalisations associated with becoming an incident senior HCU (ie, the first admission in the fiscal year when the patient reaches the HCU status) with the goal to reduce or divert them may be a more appropriate target for policy development. Since this subject has received little attention, ${ }^{24}$ more information is needed on the index hospitalisation, including the patient demographic and clinical attributes (eg, whether the patient is admitted for a newly diagnosed condition or a condition that he or she has received care in preceding years), outpatient care that was provided prior to the admission (eg, type of home care visits) and the environment within which the care is received (eg, primary care model).

Determining the most expensive conditions by inpatient costs and identifying patient attributes associated with them is also of great interest to health planners and administrators as a potential target for cost containment strategies. In this respect, the contribution of ambulatory care sensitive conditions (ACSC) to HCU requires clarification. ACSC-related hospital admissions, that is, those that are theorised to be reducible with high-quality primary care, ${ }^{25}{ }^{26}$ have been long used as an indicator of access to primary care at the population level. ${ }^{27-29}$ In Canada, several chronic ACSCs are on a national list of indicators of health system performance reported by health authorities. ${ }^{30}$ However, the economic impact of ACSC admissions among HCUs is unclear. A recent US study revealed that no more than $10 \%$ of hospitalisation costs among the top decile of Medicare HCUs were ACSC related. ${ }^{31}$ The authors commented that if the financial impact of ACSC is low and resource consumption is a target for intervention, it may be worthwhile to shift prevention efforts to other conditions that are financially more burdensome. The only Canadian study of this issue reported that $6 \%$ of hospital encounters among HCUs were considered ambulatory sensitive. This study however was different from the US study in that it defined the top 5th percentile as HCUs (versus 10th), investigated a broader population (children and adults up to 75 years of age) admitted to a single tertiary hospital in Ottawa, Ontario, and estimated the ACSC costs focusing on a shorter list of chronic conditions. ${ }^{32}$ As such, we do not know if these results are generalisable to all hospitalisations in Ontario and to the senior HCUs. None of these studies have focused on incident HCUs in which the economic impact from ACSCs may be different compared with persistent HCUs or 
on the relative contribution of ACSCs on the index hospitalisations during the incident year.

\section{Regional variation in health services use, costs and mortality among incident senior HCUs}

Finally, studying regional variation is needed to understand equality in service provision and identify areas for interventions. Evidence on geographic variation in healthcare utilisation, costs and mortality among senior HCUs is scarce. ${ }^{43}$ In Canada's general population, variation in health service use (eg, hospital admission rates, surgical procedures or consumption of medications), both at provincial level and when compared with other countries, can be substantial. ${ }^{34-37} 37$ This observation however may be misleading as assessments of variation are commonly adjusted for age and sex only ${ }^{36-39}$ despite numerous reports revealing the impact of sociodemographic or healthcare supply factors on this variation. ${ }^{35} 40-42$ However, healthcare spending may show a lower level of variability. For example, a recent study conducted in British Columbia, Canada, reported a coefficient of variation $(\mathrm{CV})$ for total healthcare spending of 8.6 (4.9 on adjustment).$^{40}$ This is lower compared with the US and the UK that reported CVs of approximately 12 and 10, respectively. ${ }^{43}$ It is unclear how all these findings relate to senior HCUs in the context of Ontario. Also, assessment of regional variation in individual cost categories has not been reported.

Furthermore, geographical units should reflect actual patterns of services use. In Ontario, delivery of care is organised by health planning regions. These regions were originally established to reflect local patterns of clinical decision making and use of services. However, inter-region migration to receive health services is common. The proportion of expenses incurred for acute care provided in health facilities outside the region of residence ranged from $3 \%$ to $49 \%$ depending on the region. ${ }^{33}$ The impact of such migration on regional variation in healthcare use and spending among HCUs has received little attention in the literature, although potential budget planning implications for health planning regions can be sizeable due to the high costs associated with HCUs.

Here, we propose to answer three inter-related research questions:

1. What is the 1-year incremental healthcare utilisation and direct financial impact on public payers of becoming an incident HCU among seniors in Ontario? Hypothesis: the greatest incremental value in utilisation and expenditures will be attributable to hospitalisation episodes followed by physician costs.

2. What are the characteristics of hospital admissions and associated costs in senior incident HCUs compared with non-HCUs in Ontario? Hypotheses: (A) causes of hospitalisation as well as individual and care factors associated with an index hospitalisation for senior HCUs differ from those of non-HCUs; and (B) the contribution of ACSCs will be high (proportion $>10 \%$ of the total hospitalisation costs) in senior HCUs and significantly higher than among non-HCUs.
3. What is the extent of regional (health planning level) variation in healthcare utilisation, costs and mortality among senior incident HCUs compared with non-HCUs in Ontario? Hypothesis: regional variation in utilisation, sector-specific costs and mortality measured by $\mathrm{CV}$ will be significantly higher in the HCU cohort than non-HCUs.

\section{METHODS AND ANALYSIS \\ Study design}

The proposed study is a retrospective population-based matched cohort study using linked administrative health data. Registration number is NCT02815930 (clinicaltrials. gov).

\section{Setting}

Ontario is the most populous province in Canada, with almost 14 million residents, representing about $40 \%$ of the Canadian population. ${ }^{44}$ It is divided into 14 Local Health Integration Networks (LHIN) that are responsible for local healthcare planning and delivery. ${ }^{45}$ The Ontario Ministry of Health and Long-Term Care (MOHLTC), using general taxation revenues $(80 \%$ provincial and $20 \%$ federal transfer), pays for approximately $70 \%$ of healthcare provided in the province. This includes 90\%-100\% funding of hospital care, physician costs, public health and prescription drugs for seniors, ${ }^{3}$ while contributions to other services (eg, long-term care facilities) are less.

\section{Study cohorts}

The study population is senior HCUs with annual total healthcare expenditures within the top 5\% threshold of all Ontarians in the fiscal year of 2013 (ie, incident year), who were not in the top 5\% in the preceding year. Total healthcare expenditures will be calculated using the Institute of Clinical Evaluative Sciences (ICES) person-level health utilisation costing algorithms. ${ }^{46}$ ICES is an independent, non-profit research corporation funded by the MOHLTC (www.ices.on.ca).

To reduce bias due to confounding, the incident HCU cohort will be matched with non-HCU in a ratio of $1: 3$ according to age at cohort entry ( \pm 1 month), sex and LHIN of patient residence. Health services utilisation and costs will be captured from 1 April 2013 to 31 March 2014.

\section{Data set}

The patient level dataset will be created using 15 health administrative databases housed at ICES. These databases contain publicly funded administrative health service records for the Ontario population eligible for health coverage. These databases are linked using encrypted patient-specific identifiers. Online supplementary appendix 1 presents a description of databases that will be used to create the dataset.

\section{Variables}

The dataset will include a number of variables related to patient sociodemographic characteristics, healthcare use 
and patient outcomes that are briefly described below (see online supplementary appendix 2 for more detail on key variables).

Patient characteristics include age, sex, geographic location, income (in quintiles), immigration status and comorbidity. Geographical location of residence (urban/suburban/ rural) is based on the Rural Index of Ontario (RIO) and LHIN. ${ }^{47}$ Multimorbidity is captured by means of John Hopkins Expanded Diagnosis Clusters (EDCs). EDCs are derived from Johns Hopkins Adjusted Clinical Groups (www.hopkinsacg.org), ${ }^{48}$ which are used to organise the codes of the International Statistical Classification of Diseases and Related Health Problems, the 10th revision, Canadian version (ICD10-CA) ${ }^{49}$ into 282 clinically similar clusters. EDCs will be based on 3 years of hospitalisation and ambulatory data prior to index date.

Care characteristics include the primary care provider payment model. The providers are categorised by several main primary care patient enrolment models: fee for service (FFS), enhanced FFS, Family Health Team (FHT), capitation and none. Under enhanced FFS model, provider's compensation is based on FFS billing with enhanced FFS components and incentives for the provision of services for specific patient needs. FHT models consists of two options: the primarily capitation-based Family Health Network (FHN) and the capitation or salaried-based Family Health Organization (FHO). If the patient is affiliated with either FHN or FHO but not matched to FHT, then the patient is placed with the capitation category. The none category refers to patients for whom no primary care provider was identified (ie, they were not enrolled with a provider through a patient enrolment programme, and they were not virtually rostered based on claims because they did not have any billing claims with primary care fee codes).

Resource utilisation variables include the number of hospitalisations, emergency department (ED) visits, physician encounters, publicly funded home care visits and long-term care. Home care visits are categorised by type of services provided such as nursing, personal support or allied health. For each hospitalisation, the following information is derived: admission type (urgent or elective), length of stay, the type of institution the patient has been transferred from, alternate level of care (ALC) status, discharge destination, date of death while in hospital, whether the hospitalisation happened within the LHIN of residence and hospitalisation costs. All healthcare expenditures are derived using the ICES costing algorithm for each cost category.

\section{Study primary outcomes}

1. One-year incremental healthcare utilisation (rate per 10000 of study population for hospital admissions, $\mathrm{ED}$, physician and home care visits) and costs (mean) attributable to becoming an HCU at the provincial level (research question 1).

2. Determination of patient and care factors associated with (A) index hospitalisation (OR) and (B) its inhospital mortality (OR) among HCUs and non-HCUs during the incident year (research question 2).
3. Proportion (\%) of ACSC-related hospitalisation costs to annual total inpatient costs during the incident year at the provincial level for the HCU and non-HCU cohorts (research question 2).

4. Patterns of variation in healthcare utilisation, mortality and costs across LHINs in HCUs compared with non-HCUs during the incident year (research question 3).

\section{ANALYSIS PLAN}

The two matched cohorts (HCUs and non-HCUs) will be described using descriptive statistics. In addition to standardised differences ${ }^{50}$ to compare the baseline characteristics of the two cohorts, regression methods will be used to adjust for important residual differences between the cohorts that remain after matching. Each subsection below presents more detail on handling confounding. Data preparation before running regression analyses will include identifying colinearity between covariates. Goodness-of-fit statistics will be used to evaluate models and guide model selection. A level of $\alpha<0.05$ will be applied to indicate statistical significance.

Regression models for cost and count data including two-part models to deal with the potential over-representation of zeros in the data will be used to analyse the data. For example, we expect that many members in the non-HCU cohort may have no encounters with the health system (ie, no hospital admission, physician visits or visits to ED). Ignoring the fact that the data are not normally distributed or using only the portion of the data with the values greater than zero can lead to biased estimates. ${ }^{51}$ The following provides more information on the analysis plan for each of the three research questions.

\section{Research question 1}

To estimate the incremental healthcare utilisation and costs attributable to becoming an HCU, longitudinal data analysis will be employed. ${ }^{52}$ The HCU dataset containing repeated measures on the same subject (ie, 1 year prevalues and postvalues) is an example of longitudinal data. Incremental values of the outcome variables (ie, costs, physician encounters and so on) represent the difference between the two cohorts over time. An estimate of incremental values will be generated using the method of recycled predictions. ${ }^{53-56}$ First, coefficients are obtained from a model regressing the postvalues of an outcome on the HCU status, prevalues of the outcome and other covariates as needed. Then, using the calculated coefficients, predicted outcome values are estimated assuming everyone is an HCU and re-estimated assuming every subject is a non-HCU. The difference between the two averaged predictions yields the incremental value. CIs of the incremental values will be obtained with the percentile method (ie, creating a bootstrap distribution and assigning the $95 \%$ lower bound CI to the 2.5th percentile and the $95 \%$ upper bound CI to the 97.5 th percentile). ${ }^{56}$ The method will be applied to analyse incremental changes in each type of costs and healthcare utilisation. 
This approach will allow us to account for correlation between the prevalues and postvalues, to adjust for residual confounding by including demographic (ie, income) and health status (ie, comorbidities) variables in the model and, when needed, to properly manage excessive zero values by developing two-part models. Alternative models may also be explored to accommodate the data specifics (eg, mixed models with random effects).

\section{Research question 2}

To describe and compare characteristics of the index hospitalisation among senior HCUs versus non-HCUs during the incident year (fiscal year 2013), we will define an index hospitalisation as the first hospitalisation in the incident year among subjects without admissions of any type in the preceding year (fiscal year 2012). We will provide descriptive statistics on hospitalisations by the type of admission (frequency of urgent vs elective), by the total length of stay (mean), including the ALC status and the number of ALC days (mean), by discharge destination (frequency) and inhospital mortality. Using major ICD10-CA diagnosis codes responsible for resource use (abbreviated as MRDX), we will identify the most common clinical causes of admissions and contrast the list with a list of most expensive hospitalised conditions for both cohorts to distinguish common diagnoses from diagnoses that drive inpatient spending. To determine patient and care factors associated with index hospitalisation and its inhospital mortality (dependent variables) during the incident year, we will develop predictive models using multivariable logistic regression based on a list of predetermined demographic, clinical and care factors (online supplementary appendix 3).

To investigate the proportion of ACSC-associated hospitalisation costs, we will identify patients admitted for ACSCs and calculate for the HCU and the non-HCU cohorts the ratio of inpatient costs incurred through ACSC admissions to the total inpatient expenditures. Our ACSC list will be based on the list originally developed by the Agency for Healthcare Research and Quality (AHRQ). ${ }^{29}$ Chronic conditions on the list (eg, hypertension, diabetes and so on) will be identified using the Canadian Institute of Health Information (CIHI) ACSC algorithm, ${ }^{27}$ which is based on the AHRQ original list adapted to Canada. The algorithm for three other conditions considered acute (eg, bacterial pneumonia, dehydration and urinary tract infections) and not included in the CIHI algorithm of chronic conditions will be derived by directly converting the original ICD-10-CM codes of the AHRQ original list into ICD-10-CA. Online supplementary appendix 4 provides more detail on the algorithms.

Consistent with the approach to ACSC identification that was previously used by researchers, ${ }^{27} 3257$ ACSC-related hospitalisations can be identified using the most responsible diagnosis at discharge. However, using the most responsible diagnosis that accounts for the largest portion of consumed resources during the hospitalisation may not be able to accurately capture all ACSC-associated admission costs. Applying an ACSC definition to preadmission diagnoses that also add to the use of resources ${ }^{57}$ would help clarify the economic impact of ACSCs among incident senior HCUs. Therefore, ACSC diagnosis codes will be included when they are accompanied by diagnosis types of either ' $\mathrm{M}$ ' (MRDX) or ' 1 ' (preadmission diagnosis) without an accompanying ' 2 ' (postadmission diagnosis) ${ }^{57}$ Of note, no studies have compared these two approaches before to identify ACSC related costs. The ACSC definition will be applied to patients in the incident year. Transfers will be excluded from the definition of hospitalisation episode.

Sensitivity analysis will be conducted to assess the impact of several factors on hospitalisation costs. Analysis will be repeated for three age subgroups: those age 66-74 years, $75-84$ years and 85 years and older. As sepsis cases (reportedly, one of the costliest among hospitalised conditions) may go under-reported when using MRDX codes alone, ${ }^{58}$ the case-finding algorithm to capture these cases will include preadmission and postadmission codes that are not MRDX. We will also apply the ACSC algorithm excluding non-emergent hospitalisations and readmissions. The ACSC-related costs will be compared with non-ACSC inpatient costs in both cohorts.

\section{Research question 3}

To assess regional differences among senior HCUs compared with non-HCUs, we will focus on the incident year and use several approaches. First, we will make a cross-sectional comparison of patients' clinical, demographic and care characteristics for each LHIN contrasting the two cohorts. Within each LIHN, urban, sub-urban and rural residence characteristics by RIO will be taken into account. Crude HCU rate per LHIN seniors will be derived to identify areas of high and low HCU incidence.

Second, we will estimate regional variation in total healthcare spending and health services utilisation and contrast these values between the two cohorts. ${ }^{59}$ Regression models with LHIN-level fixed effects will be developed using the following as dependent variables: total and sector healthcare expenditures, count data (ie, hospital admission, emergency visits, physician encounters and home care visits) and mortality. The crude values will be then adjusted to remove the influence of comorbidity, demographic and care factors or RIO status.

The magnitude of variation will be quantified using the extremal quotient (EQ), the CV and the Gini mean difference (GMD). The EQ is the ratio of the highest LHIN parameter to the lowest. The $\mathrm{CV}$ is the ratio of the $\mathrm{SD}$ to the mean among the LHINs: the higher the CV, the greater the dispersion. Both are widely used nationally and internationally. ${ }^{37} 38$ The GMD has been commonly used in economics and social sciences to measure inequality and variability and is gaining popularity in health sciences. ${ }^{60}$ It calculates the extent to which the distribution of a parameter (eg, total costs) among individuals across LHINs deviates from an exactly equal distribution.

Third, we will describe inter-LHIN migration patterns to receive acute hospital care and assess its impact on regional variation in total health spending for both cohorts. We 
will rerun the total healthcare spending regression model described above with the proportion of residents of a LHIN admitted outside the LHIN taken out of the analysis. The $\mathrm{EQ}$ the $\mathrm{CV}$ and the GMD will be used to compare the models and the cohorts.

\section{Significance and policy implications of study results}

This study will generate new knowledge that will assist Canadian healthcare administrators, clinicians, citizens and patients to guide health policy and programme development around senior HCUs. The analysis of incremental healthcare utilisation and costs will provide a description of the true utilisation and economic impact associated with the incident HCU status. By separating index hospitalisations, the analysis of hospitalisation patterns in the incident cohort of senior HCUs compared with matched non-HCUs will help identify potential interventions to prevent or divert hospitalisation episodes for high-risk groups. Exploring the contribution of disease-specific hospitalisation costs towards the total inpatient spending will help determine the potential value expanding care models that target ACSCs and identify opportunities of fund reallocation to hospitalisations types that are more contributory and more amenable to change. Furthermore, by defining regional variation in healthcare services and spending among senior HCUs, we will inform the value of potential benchmarking and regional practice comparisons in HCU management. Finally, since other jurisdictions in developed countries have comparable health systems and are faced with similar HCU challenges, our methods and findings may inform local considerations for HCU prevention and management.

\section{Exploratory analysis}

Explanatory analyses may be conducted to explore study-specific populations, cost thresholds to determine HCU status ( $1 \%$ vs $5 \%)$ or any other relevant factors. ICES-derived cohorts will be used to facilitate the analysis. These cohorts were created by identifying patients with specific diseases (eg, chronic obstructive pulmonary disease, congestive heart failure and diabetes) using validated case-finding algorithms. ${ }^{61} 62$

\section{Ethics and dissemination}

The results of the study will be distributed widely through peer-reviewed journals. They also will be disseminated at research events in academic settings, national and international conferences as well as with presentations to the MOHLTC and LHIN administration.

\footnotetext{
Author affiliations

${ }^{1}$ Department of Health Research Methods, Evidence, and Impact, McMaster University, Hamilton, Ontario, Canada

${ }^{2}$ Programs for Assessment of Technology in Health (PATH), The Research Institute of St. Joe's Hamilton, St. Joseph's Healthcare, Hamilton, Ontario, Canada

${ }^{3}$ Division of Geriatric Medicine, Department of Medicine, McMaster University, Hamilton, Ontario, Canada

${ }^{4}$ Division of Clinical Pharmacology and Toxicology, Department of Medicine, McMaster University, Hamilton, Ontario, Canada

${ }^{5}$ Geriatric Education and Research in Aging Sciences Centre, Hamilton Health Sciences, Hamilton, Ontario, Canada
}

${ }^{6}$ Institute for Clinical Evaluative Sciences (ICES), Toronto, Ontario, Canada ${ }^{7}$ Département de médecine sociale et préventive, Faculté de Médecine, Université Laval, Quebec City, Quebec, Canada

${ }^{8}$ Centre de recherche du CHU de Québec, Université Laval, Axe Santé des Populations et Pratiques Optimales en Santé, Quebec City, Quebec, Canada ${ }^{9}$ Li Ka Shing Knowledge Institute, St. Michael's Hospital, Toronto, Ontario, Canada ${ }^{10} \mathrm{Big}$ Data and Geriatric Models of Care Cluster, Department of Medicine, McMaster University, Hamilton, Ontario, Canada

${ }^{11}$ Center for Health Economics and Policy Analysis (CHEPA), McMaster University, Hamilton, Ontario, Canada

Contributors SM, J-ET, AH, JL, JMP, TG and JRG conceptualised the study. All authors have contributed to its design. JMP, WK, PP and TG were instrumental in creating datasets. SM prepared the initial draft of the manuscript and revised it based on co-authors' feedback: J-ET, AH, JL, JMP, TG, JRG, LM, APC, WK and PP provided comments to the initial draft, further revisions and read and approved the final manuscript. The responsibility of study implementation lies with the principle investigator (SM) that is supported and supervised primarily by J-ET.

Funding This work is supported by personnel funding and in-kind analyst and epidemiologist support from the Ontario Drug Policy Research Network (ODPRN) and personnel awards from the Canadian Institutes of Health Research (ClHR) Drug Safety and Effectiveness Cross-Disciplinary Training (DSECT) Program, the Program For Assessment of Technology in Health (PATH) and an Ontario Graduate Scholarship (OGS). The ODPRN is funded by grants from the Ontario Ministry of Health and Long-Term Care (MOHLTC) and Ontario Strategy for Patient-Orientated Research (SPOR) Support Unit, which is supported by the Canadian Institutes of Health Research and the Province of Ontario. The opinions, results and conclusions reported in this article are those of the authors and are independent from the funding sources. No endorsement by the Ontario MOHLTC is intended or should be inferred.

Competing interests None declared.

Ethics approval This study has been approved by Hamilton Integrated Research Ethics Board (ID\#1715-C).

Provenance and peer review Not commissioned; externally peer reviewed.

Open Access This is an Open Access article distributed in accordance with the Creative Commons Attribution Non Commercial (CC BY-NC 4.0) license, which permits others to distribute, remix, adapt, build upon this work non-commercially, and license their derivative works on different terms, provided the original work is properly cited and the use is non-commercial. See: http://creativecommons.org/ licenses/by-nc/4.0/

(c) Article author(s) (or their employer(s) unless otherwise stated in the text of the article) 2017. All rights reserved. No commercial use is permitted unless otherwise expressly granted.

\section{REFERENCES}

1. World population ageing 2013: United Nations, Department of Economic and Social Affairs, Population Division, 2013.

2. Institute for Research on Public Policy. 2015. Designing a national seniors strategy for Canada, IRPP task force on aging: Institute for Research on Public Policy.

3. Canadian Institute for Health Information. 2016. National health expenditure trends, 1975 to 2016. Ottawa: Canadian Institute for Health Information.

4. Rais S, Nazerian A, Ardal S, et al. High-cost users of Ontario's healthcare services. Healthc Policy 2013;9:44-51.

5. Rosella LC, Fitzpatrick T, Wodchis WP, et al. High-cost health care users in Ontario, Canada: demographic, socio-economic, and health status characteristics. BMC Health Serv Res 2014;14:532.

6. Central Midlands CSU NHS. The total cost of care for older people in Solihull - an analysis of combined NHS and social care data. 2014 https://solihullccg.nhs.uk/publications/1245-bcf-cost-of-care/file (accessed 20 May 2017).

7. Stanton MW RM. High concentration of US health expenditures: Research in Action, Agency for Healthcare Research and Quality, 2006.

8. Wodchis WP, Austin PC, Henry DA. A 3-year study of high-cost users of health care. CMAJ 2016;188:182-8.

9. Sinha SK. Why the elderly could bankrupt Canada and how demographic imperatives will force the redesign of acute care service delivery. Healthc Pap 2011;11:46-51. 
10. Holtz-Eakin D. High-cost medicare beneficiaries 2005. 2005 http:// www.cbo.gov/publication/16487 (accessed 25 Apr 2017).

11. Nelson L. Lessons from medicare's demonstration projectson disease management and care coordination. Washington, DC: Health and Human Resources Division Congressional Budget Office, 2012.

12. Peikes D, Chen A, Schore J, et al. Effects of care coordination on hospitalization, quality of care, and health care expenditures among Medicare beneficiaries: 15 randomized trials. JAMA 2009;301:603-18.

13. Roos NP, Shapiro E, Tate R. Does a small minority of elderly account for a majority of health care expenditures? A sixteen-year perspective. Milbank Q 1989;67:347-69.

14. Blumenthal D, Chernof B, Fulmer T, et al. Caring for high-need, highcost patients - an urgent priority. N Engl J Med 2016;375:909-11.

15. Stokes J, Panagioti M, Alam R, et al. Effectiveness of case management for 'At Risk' patients in primary care: a systematic review and meta-analysis. PLoS One 2015;10:e0132340.

16. Fitzpatrick T, Rosella LC, Calzavara A, et al. Looking beyond income and education: socioeconomic status gradients among future highcost users of health care. Am J Prev Med 2015;49:161-71.

17. Reid R, Evans R, Barer M, et al. Conspicuous consumption: characterizing high users of physician services in one Canadian province. J Health Serv Res Policy 2003;8:215-24.

18. Guilcher SJ, Bronskill SE, Guan J, et al. Who are the high-cost users? A method for person-centred attribution of health care spending. PLOS One 2016;11:e0149179.

19. Dattalo M, Nothelle S, Chapman EN. Targeting enhanced services toward high-cost, high-need medicare patients. 2016:19-30.

20. Iloabuchi TC, Mi D, Tu W, et al. Risk factors for early hospital readmission in low-income elderly adults. J Am Geriatr Soc 2014:62:489-94.

21. Goncalves-Bradley DC, Lannin NA, Clemson LM, et al. Discharge planning from hospital. The Cochrane database of systematic reviews 2016;1:Cd000313.

22. Kellogg FR, Brickner PW, Conley L, et al. Controlling hospital readmission of elderly persons living at home: a risk factor analysis. Home Health Care Serv Q 1991;12:5-16.

23. White Paper, Health Care Transformation Task Force. Health care transformation task force. $2015 \mathrm{http}: / / \mathrm{hcttf} .0 \mathrm{rg} /$ resources-toolsarchive/2015/5/14/whitepaper (accessed 10 Mar 2017).

24. Wallace E, Stuart E, Vaughan N, et al. Risk prediction models to predict emergency hospital admission in community-dwelling adults: a systematic review. Med Care 2014;52:751-65.

25. Ministry of Health and Long Term Care. Health system information management \& investment division. Health analytics branch. http:// www.health.gov.on.ca/en/pro/programs/ris/docs/ambulatory_care_ sensitive_conditions.pdf (accessed 20 Nov 2016).

26. Billings J, Zeitel L, Lukomnik J, et al. Impact of socioeconomic status on hospital use in New York City. Health Aff 1993;12:162-73.

27. Canadian Institute for Health Information. Ambulatory care sensitive conditions, 2016. http://indicatorlibrary.cihi.ca/display/HSPIL/ Ambulatory+Care+Sensitive+Conditions

28. Longman JM, Passey ME, Ewald DP, et al. Admissions for chronic ambulatory care sensitive conditions - a useful measure of potentially preventable admission? BMC Health Serv Res 2015;15:472.

29. Agency for Healthcare Research and Quality. AHRQ Quality Indicators - prevention quality indicators technical specifications updates. Version 6.0 (ICD $10 \mathrm{http}: / /$ www.qualityindicators.ahrq.gov/ Modules/PQI TechSpec ICD10 v60.aspx 2016.

30. Ontario South West Local Health Integration Network. Report on performance: technical specifications. $2017 \mathrm{http}: / / \mathrm{www}$. southwestlhin.on.ca/accountability/Performance.aspx\#Big\%20Dot\% 20and\%20Priority\%20Summaries

31. Joynt KE, Gawande AA, Orav EJ, et al. Contribution of preventable acute care spending to total spending for high-cost Medicare patients. JAMA 2013;309:2572-8.

32. Ronksley PE, Kobewka DM, McKay JA, et al. Clinical characteristics and preventable acute care spending among a high cost inpatient population. BMC Health Serv Res 2016;16:165.

33. Health Analytics Branch, HSIMI. High cost users for local health integrated networks. 2013 http://southwestlhin.on.ca/ /media/sites/ sw/uploadedfiles/Public Community/Current Initiatives/Health Links/Describing\%20High\%20Users_HAB_overview_September\% 202013\%20

34. Corallo AN, Croxford R, Goodman DC, et al. A systematic review of medical practice variation in OECD countries. Health Policy 2014:114:5-14.

35. Hogan DB, Maxwell CJ, Fung TS, et al. Regional variation in the use of medications by older Canadians--a persistent and incompletely understood phenomena. Pharmacoepidemiol Drug Saf 2003;12:575-82.

36. Feinberg $\mathrm{AE}$, Porter $\mathrm{J}$, Saskin $\mathrm{R}$, et al. Regional variation in the use of surgery in Ontario. CMAJ Open 2015;3:E310-E316.

37. Geographic Variations in Health Care. Focus on health- OECD health policy studies. $2014 \mathrm{https} / / / w w w . o e c d . o r g / e l s /$ health-systems/ FOCUS-on-Geographic-Variations-in-Health-Care.pdf (accessed 18 May 2017)

38. Feasby TE, Quan H, Ghali WA. Geographic variation in the rate of carotid endarterectomy in Canada. Stroke 2001;32:2417-22.

39. Kim AM, Park JH, Kang S, et al. The effect of geographic units of analysis on measuring geographic variation in medical services utilization. J Prev Med Public Health 2016;49:230-9.

40. Lavergne MR, Barer M, Law MR, et al. Examining regional variation in health care spending in British Columbia, Canada. Health Policy 2016;120:739-48.

41. Kephart GAY, Atherton $\mathrm{F}$, Burge $\mathrm{F}$, et al. Small area variation in rates of high-cost healthcare use across Nova Scotia. Halifax, Nova Scotia: Maritime SPOR SUPPORT Unit, 2016.

42. Morgan SG, Cunningham CM, Hanley GE. Individual and contextual determinants of regional variation in prescription drug use: an analysis of administrative data from British Columbia. PLoS One 2010;5:e15883.

43. Congressional Budget Office, Congress of The United States. Geographic variation in health care spending. $2008 \mathrm{https}: / / \mathrm{www}$. cbo.gov/sites/default/files/cbofiles/ftpdocs/89xx/doc8972/02-15geoghealth.pdf (accessed 8 Jun 2017).

44. Statistics Canada. Population by year, by province and territory. 2016 http://www.statcan.gc.ca/tables-tableaux/sum-som/l01/cst01/ demo02a-eng.htm (accessed 26 Apr 2017)

45. Office of the Audit or General of Ontario. 2015. Annual report 2015: Office of the Audit or General of Ontario.

46. Wodchis WP, Bushmeneva K, Nikitovic M, et al. Guidelines on person-level costing using administrative databases in Ontario1. Working Paper Series 2013.

47. Kralj B. Measuring 'rurality' for purposes of health-care planning: an empirical measure for Ontario: Ontario Medical Review, 2000.

48. The Johns Hopkins ACG® system version 11.0 technical reference guide: Department of Health Policy and Management, Johns Hopkins University, Bloomberg School of Public Health, 2014.

49. Canadian Institute for Health Information. ICD-10-CA (International statistical classification of diseases and related health problems, 10th Revision, Canada). (accessed 25 May 2017).

50. Austin PC. Using the standardized difference to compare the prevalence of a binary variable between two groups in observational research. Commun Stat Simul Comput 2009;38:1228-34.

51. Tooze JA, Grunwald GK, Jones RH. Analysis of repeated measures data with clumping at zero. Stat Methods Med Res 2002;11:341-55.

52. Diggle PJ HP, Liang K-Y, Zeger SL. Analysis of longitudinal data. 2nd edn. Clarendon: Oxford University Press Inc, 2002.

53. Basu A, Arondekar BV, Rathouz PJ. Scale of interest versus scale of estimation: comparing alternative estimators for the incremental costs of a comorbidity. Health Econ 2006;15:1091-107.

54. Chang C, Lee SM, Choi BW, et al. Costs attributable to overweight and obesity in working asthma patients in the United States. Yonsei Med J 2017:58:187-94.

55. Lange A, Zeidler J, Braun S. One-year disease-related health care costs of incident vertebral fractures in osteoporotic patients. Osteoporos Int 2014;25:2435-43.

56. Mannino DM, Higuchi K, Yu TC, et al. Economic Burden of COPD in the presence of comorbidities. Chest 2015;148:138-50.

57. Walker JD, Teare GF, Hogan DB, et al. Identifying potentially avoidable hospital admissions from canadian long-term care facilities. Med Care 2009:47:250-4.

58. Rhee C, Dantes R, Epstein L, et al. Incidence and trends of sepsis in US hospitals using clinical vs claims data, 2009-2014. JAMA 2017:318:1241-9.

59. Austin P, Merlo J. Intermediate and advanced topics in multilevel logistic regression analysis, 2017.

60. Gadrich T, Bashkansky E, Zitikis R. Assessing variation: a unifying approach for all scales of measurement. Qual Quant 2015;49:1145-67.

61. Gershon AS, Wang C, Guan J, et al. Identifying individuals with physcian diagnosed COPD in health administrative databases. COPD 2009;6:388-94.

62. Schultz SE, Rothwell DM, Chen Z, et al. Identifying cases of congestive heart failure from administrative data: a validation study using primary care patient records. Chronic Dis Inj Can 2013;33:160-6. 